\title{
MECANISMO DE EVOLUÇÃO DE HIDROGÊNIO DURANTE A CORROSÃO DA LIGA 2198-T8 EM MEIO CONTENDO CLORETO: UMA ABORDAGEM ELETROQUÍMICA
}

\author{
João Victor de Sousa Araujo*,a, , Rejane Maria Pereira da Silva ${ }^{a}$, Mariana Xavier Milagre ${ }^{a}$, Caruline de Souza Carvalho \\ Machado $^{\mathrm{a}}$ e Isolda Costa ${ }^{\mathrm{a}}$ \\ ${ }^{a}$ Centro de Ciência e Tecnologia de Materiais, Instituto de Pesquisas Energéticas e Nucleares, Universidade de São Paulo, 05508-000 \\ São Paulo- SP, Brasil
}

Recebido em 27/03/2020; aceito em 11/06/2020; publicado na web em 24/07/2020

\begin{abstract}
ON THE HYDROGEN EVOLUTION MECHANISM DURING CORROSION OF THE 2198-T8 ALLOY IN CHLORIDE SOLUTION: AN ELECTROCHEMICAL APPROACH. Corrosion is still one of the most serious and frequent problems in industries. The phenomena involved in the corrosion mechanism of Al-alloys may be explained by formation of galvanic cells between the metal matrix and heterogeneities, such as precipitates, intermetallic phases leading to potential differences and electrochemical reactions. In the cathodic sites, the main reactions are oxygen reduction reaction, in aerated neutral environments, and hydrogen evolution in acid media. This last type of reaction might also occur in neutral solutions inside pits (anodic region). In this study, the mechanism of hydrogen evolution during corrosion of the 2198-T8 Al-Cu-Li alloy exposed in a chloride solution was investigated. The mechanism was related to the presence of $\mathrm{T} 1$ phase $\left(\mathrm{Al}_{2} \mathrm{CuLi}\right)$, which is the main strengthening phase in this material. This phase is highly active and, when exposed to corrosive media, leads to severe localized corrosion (SLC). One of the main characteristics related to SLC is hydrogen gas evolution which was confirmed by gel visualization. In this study, the hydrogen evolution mechanism inside the SLC sites was studied by scanning electrochemical microscopy (SECM).
\end{abstract}

Keywords: Al-Cu-Li alloy; severe localized corrosion; hydrogen evolution; SECM.

\section{INTRODUÇÃO}

A corrosão é um processo de degradação que ocorre nos materiais metálicos, sendo a mesma causada pela oxidação de átomos da superfície dos metais. ${ }^{1}$ A Figura 1 mostra as reações típicas de corrosão em uma interface metal-água.

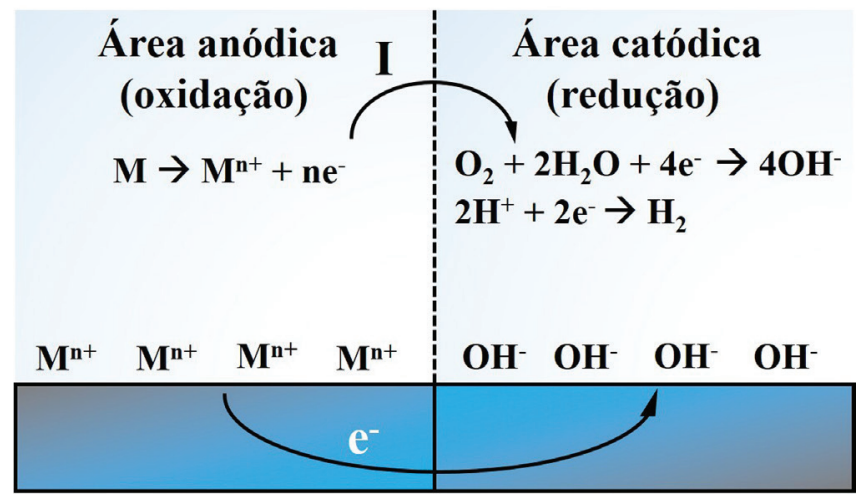

Figura 1. Ilustração esquemática mostrando as reações típicas de corrosão

As reações envolvidas no processo de corrosão levam a um aumento na densidade de cátions (não necessariamente íons metálicos) e à liberação de elétrons na interface eletrodo / eletrólito. ${ }^{1}$ Para o progresso contínuo da oxidação, os elétrons produzidos são transferidos para a área interfacial onde ocorre a redução, área catódica (Figura 1). Normalmente, isso corresponde à redução de $\mathrm{O}_{2}$ ou $\mathrm{H}^{+}$, levando a um aumento na densidade de ânions (por exemplo, $\mathrm{OH}^{-}$) ou a uma diminuição na densidade de cátions (por exemplo, $\mathrm{H}^{+}$) na interface eletrodo/eletrólito. ${ }^{1}$ Os pares de áreas anódicas e catódicas são referidos como células de corrosão e exercem um papel importante neste processo. ${ }^{2}$ A fim de reduzir perdas econômicas causadas pela corrosão, as quais correspondem a cerca de $4 \%$ do PIB, um grande esforço tem sido feito na tentativa de prever, entender e prevenir a corrosão. ${ }^{3}$ Exemplos incluem a corrosão do aço inoxidável em biomateriais, ${ }^{4}$ em instalações químicas, ${ }^{5}$ em dispositivos semicondutores, ${ }^{6}$ e nos materiais utilizados na indústria aeroespacial. ${ }^{7}$ Nesse último, destacam-se as ligas Al-Cu-Li que são utilizadas na fabricação de aeronaves como, por exemplo, na fuselagem da aeronave Airbus A350. ${ }^{8}$ Trabalhos recentes estudaram o desenvolvimento de corrosão localizada neste tipo de ligas. ${ }^{9-12}$ Em alguns destes trabalhos foi relatada a evolução de gás hidrogênio da superfície do material, quando imerso em solução corrosiva; contudo, poucos autores explicaram detalhadamente este fenômeno. ${ }^{13-15}$ Chen et al. ${ }^{13}$ estudaram a resistência à corrosão de uma liga Al-Cu-Li (2099) por meio de ensaios de imersão e técnicas eletroquímicas globais na presença de íons cloreto. Os autores observaram, durante os testes, a evolução de gás hidrogênio na superfície da liga. Contudo, um mecanismo para o fenômeno não foi proposto. Ma et al. ${ }^{14} \mathrm{em}$ um estudo realizado com a mesma liga, visualizaram a evolução de hidrogênio através de imagens de vídeo durante o teste de imersão em diferentes períodos em solução 3,5\% $\mathrm{NaCl}$. Recentemente, Lei et al. ${ }^{15}$ também verificaram a evolução de hidrogênio a em uma liga $\mathrm{Al}-\mathrm{Cu}-\mathrm{Li}$. Apesar desse mecanismo ser reportado na literatura, ${ }^{13-15}$ nesses estudos não foram utilizadas técnicas eletroquímicas locais, o que é de grande importância para investigação dos processos de corrosão em ligas de Al. ${ }^{10,16}$ Nesse contexto, este trabalho visa contribuir na compreensão eletroquímica do mecanismo de evolução de hidrogênio em uma liga $\mathrm{Al}-\mathrm{Cu}-\mathrm{Li}$ quando exposta a um meio agressivo. Para tanto uma correlação direta entre a microestrutura e os mecanismos de corrosão associados foi estabelecida por meio do monitoramento do processo de corrosão e da utilização da técnica eletroquímica local. 


\section{PARTE EXPERIMENTAL}

\section{Material}

Neste trabalho foi estudada a liga 2198-T8, cuja composição química corresponde, em \% em massa: 94,6 Al, 3,7 Cu, 1,0 Li, 0,3 Mg, 0,2 Ag, 0,12 Zr, 0,04 Fe, 0,03 Si, 0,03 Zn, e 0,003 Mn. A designação T8 indica que a liga foi deformada e envelhecida artificialmente durante o seu processo de fabricação. ${ }^{17}$ Segundo a literatura, nesse procedimento a liga é submetida a 3,5\% de deformação e o envelhecimento artificial ocorre na faixa de temperatura de $150-250{ }^{\circ} \mathrm{C}$ durante 5 -10 horas. ${ }^{18}$

\section{Caracterização microestrutural}

Amostras da liga 2198-T8 foram cortadas na seção longitudinal em relação à direção de deformação para caracterização microestrutural e para os ensaios de corrosão. Após o corte, as amostras foram embutidas em resina de cura a frio. Em seguida, as amostras foram lixadas com lixas de $\mathrm{SiC}$ (até a granulometria P4000) e polidas mecanicamente com suspensões de diamante de $3 \mu \mathrm{m}$ e $1 \mu \mathrm{m}$. Uma solução composta por $2 \% \mathrm{HF}$ e $25 \% \mathrm{HNO}_{3}$ em água destilada foi utilizada para ataque metalográfico. ${ }^{19} \mathrm{~A}$ superfície atacada foi observada em microscópio ótico (MO) Leica, Modelo DMLM. Para caracterização das partículas constituintes, bem como sua morfologia e composição química, foi utilizada a microscopia eletrônica de varredura acoplado a espectroscopia por energia dispersiva (MEVEDS). O equipamento utilizado foi o Modelo JSM-6701F, marca Jeol, com EDS acoplado com limite de detecção da ordem de $1 \%(\mathrm{~m} / \mathrm{m})$ (com penetração de feixe e resolução lateral estimada da ordem de $1 \mu \mathrm{m}$ a $3 \mu \mathrm{m})$. As imagens foram obtidas de amostras polidas sem nenhum tipo de ataque. Para caracterização dos precipitados endurecedores, de escala nanométrica, foi utilizado microscópio eletrônico de transmissão (MET), modelo JEM-2100, operando a $200 \mathrm{kV}$. As amostras analisadas por MET foram preparadas por lixamento com papel de $\mathrm{SiC}$ de granulometria P1200 até atingirem espessura média de $100 \mu \mathrm{m}$. Em seguida, foram preparados discos de $3 \mathrm{~mm}$ de diâmetro por processo de eletroerosão. Subsequentemente, as amostras foram lixadas até os discos atingirem espessuras que permitissem o polimento eletrolítico, o qual foi realizado no equipamento Electromed-4. Uma placa de aço inoxidável foi utilizada como cátodo, e o eletrólito foi composto por uma mistura de $700 \mathrm{~mL}$ metanol, $120 \mathrm{~mL}$ de água destilada, com voltagem mantida em $37 \mathrm{~V}$ e temperatura de $38^{\circ} \mathrm{C}$ por 45 segundos..$^{20}$

\section{Ensaios de corrosão}

\section{Teste de imersão}

A fim de avaliar a propagação da corrosão localizada em função do tempo, o teste de imersão com a amostra da liga estudada foi realizado em solução de $0,01 \mathrm{~mol} \mathrm{~L}^{-1} \mathrm{NaCl}$. O tempo total de ensaio foi de $24 \mathrm{~h}$, porém, a cada $2 \mathrm{~h}$ as amostras foram removidas da solução, lavadas em água deionizada, secadas e examinadas, a fim de observar a evolução da corrosão na superfície. A caracterização das amostras após o teste de imersão foi realizada por MEV usando o equipamento HITACHI TM3000. As superfícies das amostras utilizadas no teste de imersão também foram analisadas por perfilometria ótica utilizando o equipamento ZYGO's ZeGage ${ }^{\mathrm{TM}}$, a fim de verificar a profundidade do ataque localizado.

\section{Teste de gelificação}

O teste de gelificação é uma análise qualitativa que tem como objetivo observar o desenvolvimento do $\mathrm{pH}$ na superfície das amostras em consequência da corrosão, quando expostas a meio agressivo.
Neste estudo, as amostras foram expostas à solução $0,6 \mathrm{~mol} \mathrm{~L}^{-1}$ $\mathrm{NaCl}, 3 \mathrm{~g}$ de agar-agar e $7 \mathrm{~mL}$ de indicador universal em $100 \mathrm{~mL}$ de $\mathrm{H}_{2} \mathrm{O}$ aquecidos a $100{ }^{\circ} \mathrm{C}$. A concentração de $\mathrm{NaCl}$ deste ensaio foi aumentada em relação ao ensaio de imersão, com o objetivo de acelerar os processos corrosivos. Neste ensaio, ${ }^{21} \mathrm{em}$ decorrência da hidrólise promovida pelos íons de alumínio (reação 1), as regiões anódicas do gel assumem a cor laranja. Nas regiões catódicas, por sua vez, ocorre a formação de íons hidroxila, em consequência da reação de redução do oxigênio (reação 2), resultando no aparecimento da coloração verde/azulada.

$$
\begin{gathered}
\mathrm{Al}^{3+}+\mathrm{H}_{2} \mathrm{O} \rightarrow \mathrm{Al}(\mathrm{OH})^{2+}+\mathrm{H}^{+} \\
\mathrm{O}_{2}+2 \mathrm{H}_{2} \mathrm{O}+4 \mathrm{e}^{-} \rightarrow 4 \mathrm{OH}^{-}
\end{gathered}
$$

\section{Ensaios eletroquímicos}

Microscopia eletroquímica de varredura (SECM)

A microscopia eletroquímica de varredura (SECM) é uma técnica de varredura de alta resolução, utilizada para monitorar processos de corrosão in situ por meio da detecção localizada de espécies eletroquimicamente ativas em diferentes superficies. ${ }^{22-25} \mathrm{~A}$ observação da superfície por SECM foi realizada usando microscópio Sensolytics ( $\mathrm{GmbH}$ Bochum). Os ensaios eletroquímicos foram realizados em potencial de circuito aberto utilizando arranjo experimental de três eletrodos, com um microeletrodo de de Pt (tip) de $25 \mu \mathrm{m}$ de diâmetro, como eletrodo de trabalho; $\mathrm{Ag} / \mathrm{AgCl}_{\mathrm{KCl}(\text { sat) }}$ como eletrodo de referência, e fio de platina como contra eletrodo. A área superficial das amostras exposta ao meio corrosivo foi de $18 \mathrm{~mm}^{2}$. As análises foram feitas em alturas selecionadas acima da superfície da amostra após a obtenção das curvas de aproximação (no eixo z), com a ponta posteriormente ajustada a $20 \mu \mathrm{m}$ acima da superfície. A essa distância, foi possível observar variação no perfil da corrente limite, próxima à superfície, com o microeletrodo registrando a resposta, em termos de atividade, da superfície. As imagens de SECM foram obtidas após varredura do microeletrodo no plano xy, registrando a corrente da ponta em função da sua localização. Os experimentos foram realizados utilizando o modo SECM, geração no substrato, e coleta no microeletrodo (GS/CM). No modo GS/CM o microeletrodo é mantido num potencial onde a reação de eletrodo ocorre e o produto da reação no substrato, então, pode ser coletado. Assim, no modo GS/CM, o $\mathrm{H}_{2}$ resultante das atividades de corrosão na superfície da amostra é detectado como reação de oxidação na ponta, como mostra a Figura 2:

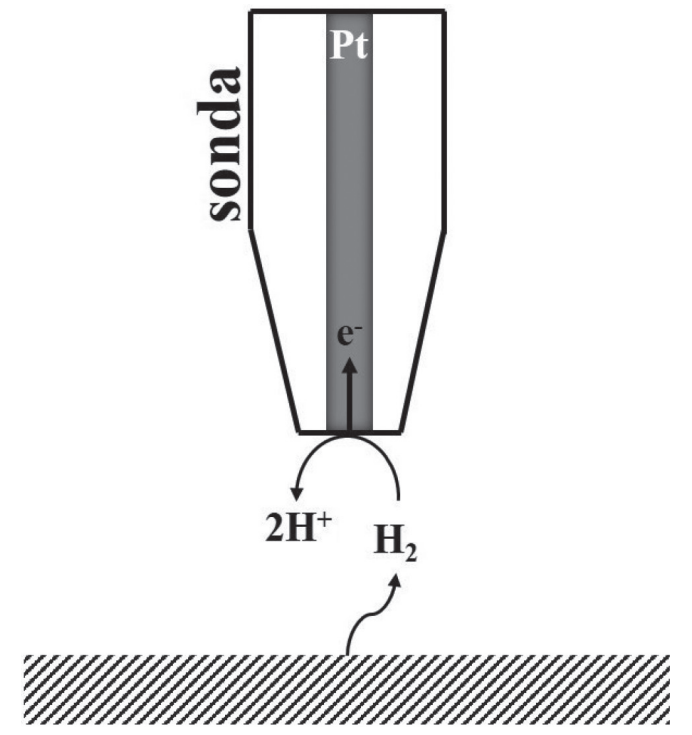

Figura 2. Representação esquemática do SECM no modo de operação GS/SM 
$\mathrm{O}_{2}$ gerado pode ser detectado em uma reação de oxidação $\left(\mathrm{H}_{2} \rightarrow 2 \mathrm{H}^{+}+2 \mathrm{e}^{-}\right)$no microeletrodo de $\mathrm{Pt}$ quando este é polarizado no potencial de $0,0 \mathrm{~V}$ versus $\mathrm{Ag} / \mathrm{AgCl}$.

\section{RESULTADOS E DISCUSSÃO}

\section{Caracterização microestrutural}

As principais características microestruturais da liga 2198-T8 são mostradas na Figura 3.

A Figura 3a mostra a morfologia dos grãos da superfície da liga em estudo. É possível observar que os mesmos estão alongados na direção da deformação, como indica a flexa branca. Esta característica é comum a materiais que foram extrudados ou laminados, ocasionando assim uma textura cristalográfica na direção preferencial de deformação. ${ }^{26}$ A microestrutura desses materiais também é composta por partículas micrométricas, como mostra a Figura $3 b$, as quais são formadas durante a fundição devido à baixa solubilidade de elementos de liga, principalmente o $\mathrm{Fe}$ no $\mathrm{Al} .{ }^{27}$ Assim, tais partículas são compostas principalmente de $\mathrm{Al}, \mathrm{Cu}$ e $\mathrm{Fe},{ }^{28}$ como indica o espectro na Figura 3c. Nas ligas Al-Cu-Li é comum encontrar fases nanométricas que atuam como partículas endurecedoras, ou seja, aumentam a resistência mecânica do material. ${ }^{7}$ A fase nanométrica $\mathrm{T} 1\left(\mathrm{Al}_{2} \mathrm{CuLi}\right)$ apresenta morfologia de plaquetas hexagonais finas ${ }^{29}$ como mostra a Figura $3 \mathrm{~d}$, e é conhecida como a principal fase endurecedora destas ligas. ${ }^{7}$ Sua nucleação ocorre preferencialmente nos defeitos cristalográficos, tais como discordâncias, contornos de grão e contornos de subgrãos. ${ }^{7}$

\section{Ensaios de corrosão}

Como observado na Figura 3, a microestrutura da liga é composta pelas partículas micrométricas (PM) e os precipitados endurecedores nanométricos. Ambos têm influência significativa no seu comportamento de corrosão, pois, atuam como pilhas de ação local que desencadeiam reações eletroquímicas responsáveis pela propagação do ataque, como mostrado na Figura 1 . O monitoramento da superfície da liga estudada exposta a solução contendo $0,01 \mathrm{~mol} \mathrm{~L}^{-1} \mathrm{NaCl} \mathrm{em}$ função do tempo, é mostrado na Figura 4.
Como observado, duas formas de corrosão, a saber comuns as ligas de $\mathrm{Al},{ }^{30,31}$ cavidades associadas às partículas micrométricas (PM), e corrosão localizada severa (CLS), relacionada aos precipitados endurecedores,$^{28}$ foram encontrados. Esses dois tipos de ataque foram observados desde as primeiras horas de imersão. $\mathrm{O}$ ataque associado às PM tem como principal característica o ataque ao redor das partículas. O processo se desenvolve devido à formação de micro células galvânicas onde as partículas atuam como áreas catódicas em relação à matriz, resultando na formação de cavidades ou trincheiras (trenching). ${ }^{28}$ Além disso, observou-se com decorrer do teste de imersão a formação de produtos de corrosão nas áreas associadas à CLS, como mostra a Figura 5.

$\mathrm{O}$ produto de corrosão formado durante a corrosão do $\mathrm{Al}$, Figura $5 \mathrm{c}$, é composto por $\mathrm{Al}(\mathrm{OH})_{3}$ e possue uma característica gelatinosa. ${ }^{32}$ Em maiores magnificações também foi possível observar as diferenças na morfologia dos ataques associados às partículas micrométricas e à CLS, como mostra a Figura 6.

Os ataques associados às partículas micrométricas foram distribuídos de maneira uniforme na superfície exposta da liga, como observado na Figura 6a-6b, ao contrário da CLS, que mostra uma propagação bem definida. A flecha amarela na Figura $6 \mathrm{c}$ indica a orientação do ataque localizado, associado ao ataque severo. É possível observar que este ataque segue a mesma orientação dos grãos, Figura 3a. A CLS está associada com a alta eletroatividade da fase T1 $\left(\mathrm{Al}_{2} \mathrm{CuLi}\right)$ concentrada em grãos com maior nível de deformação. ${ }^{33}$ Por esse motivo, durante a deformação plástica (processamento industrial) estes grãos são mais deformados do que os outros e, consequentemente, o número de discordâncias e subgrãos aumentam. Durante o envelhecimento artificial, a cinética de nucleação da fase T1 é influenciada pela energia dos defeitos. Sendo assim, grãos mais deformados têm maior densidade de discordâncias (defeitos) e, consequentemente maior fração volumétrica da fase T1.$^{34}$ Quando a superfície da liga é exposta a um ambiente corrosivo, a corrosão localizada se desenvolve preferencialmente nos grãos mais deformados e se propaga ao longo dos subgrãos, gerando ataque no interior do grão, como mostra a Figura 5a e 5b. Durante os ensaios de imersão foi observada formação de bolhas nas regiões do entorno da CLS, como mostra a Figura 7.
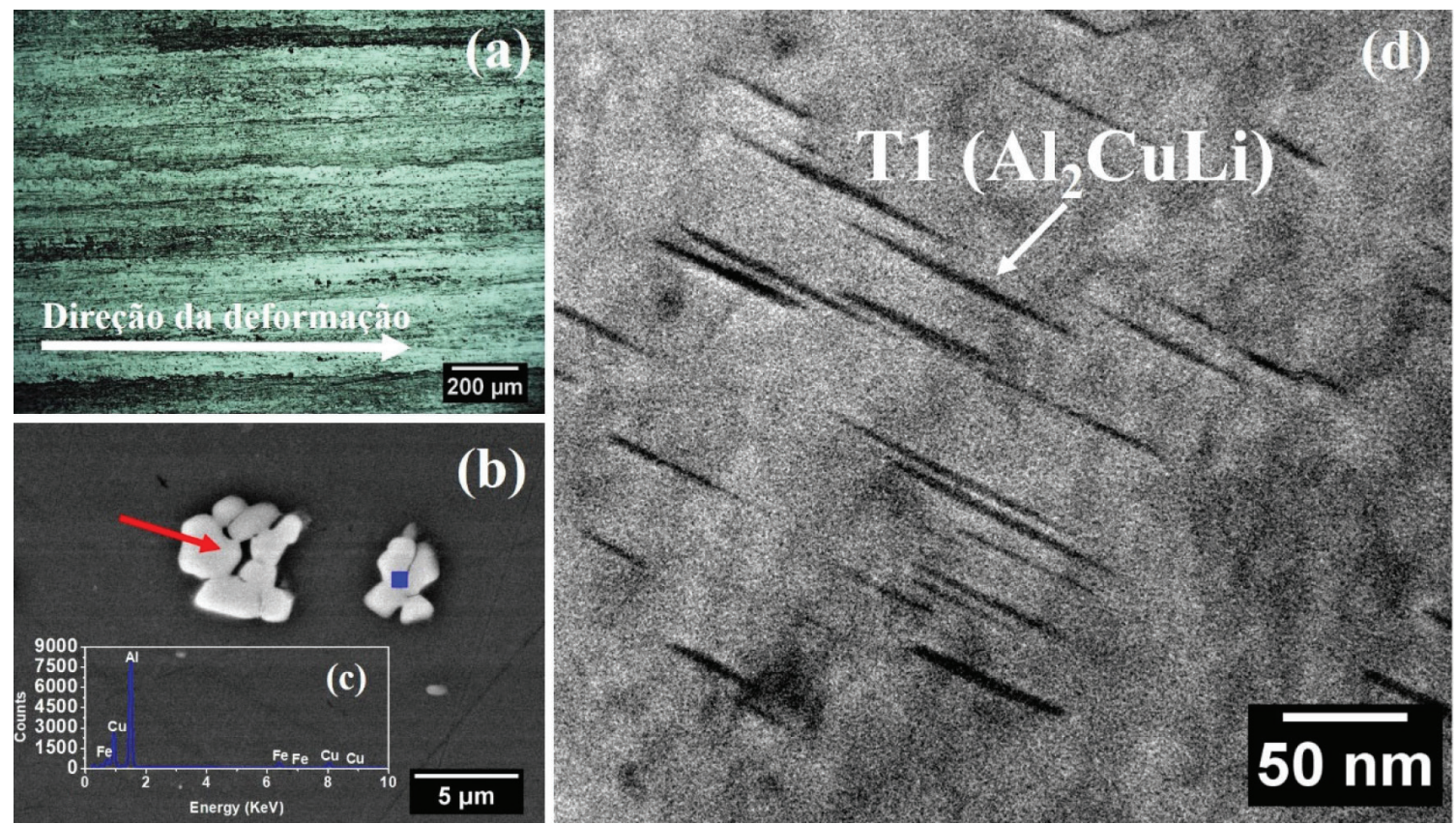

Figura 3. Características microestruturais da liga 2198-T8: (a) micrografia obtida por microscopia ótica da superfície; (b) micrografia obtida por MEV evidenciando as partículas micrométricas e (c) espectro de EDS da partícula indicada em (b); (d) micrografia por TEM mostrando a fase T1 

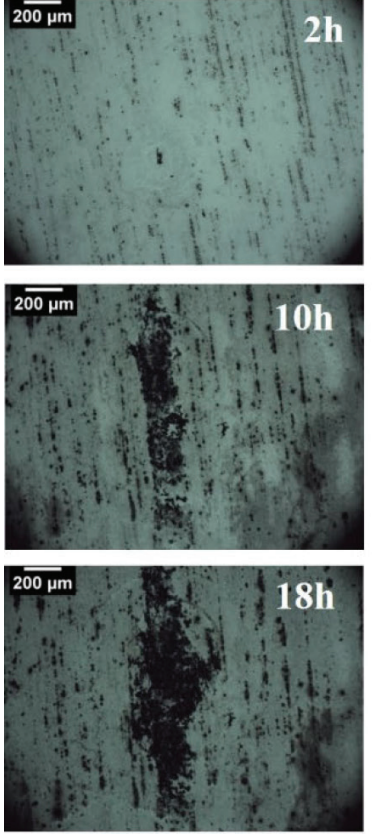
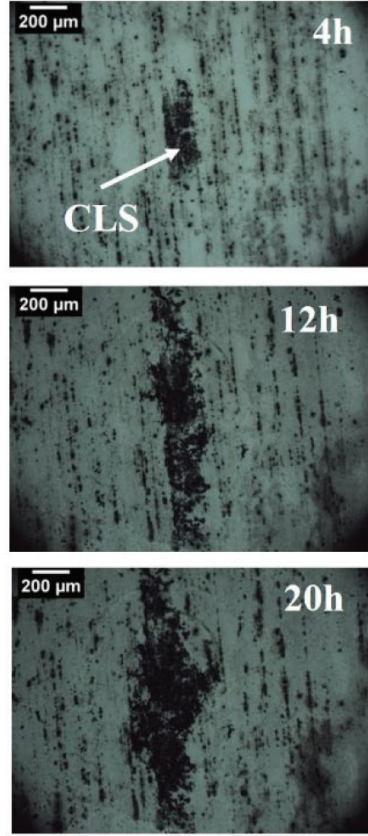
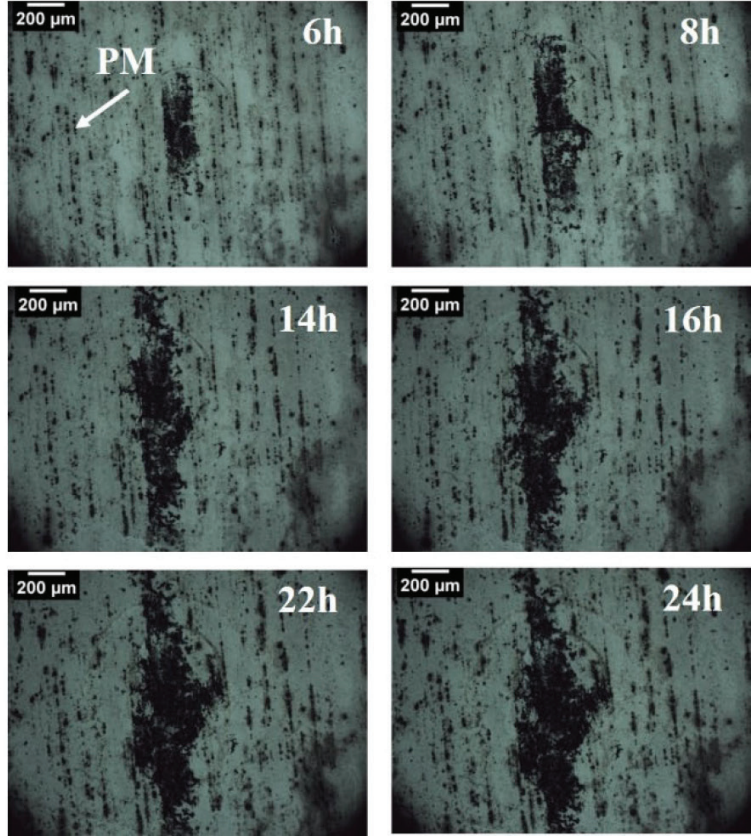

Figura 4. Micrografias óticas da superfície da liga 2198-T8 após vários períodos de exposição à solução 0,01 mol $\mathrm{L}^{-1} \mathrm{NaCl}$

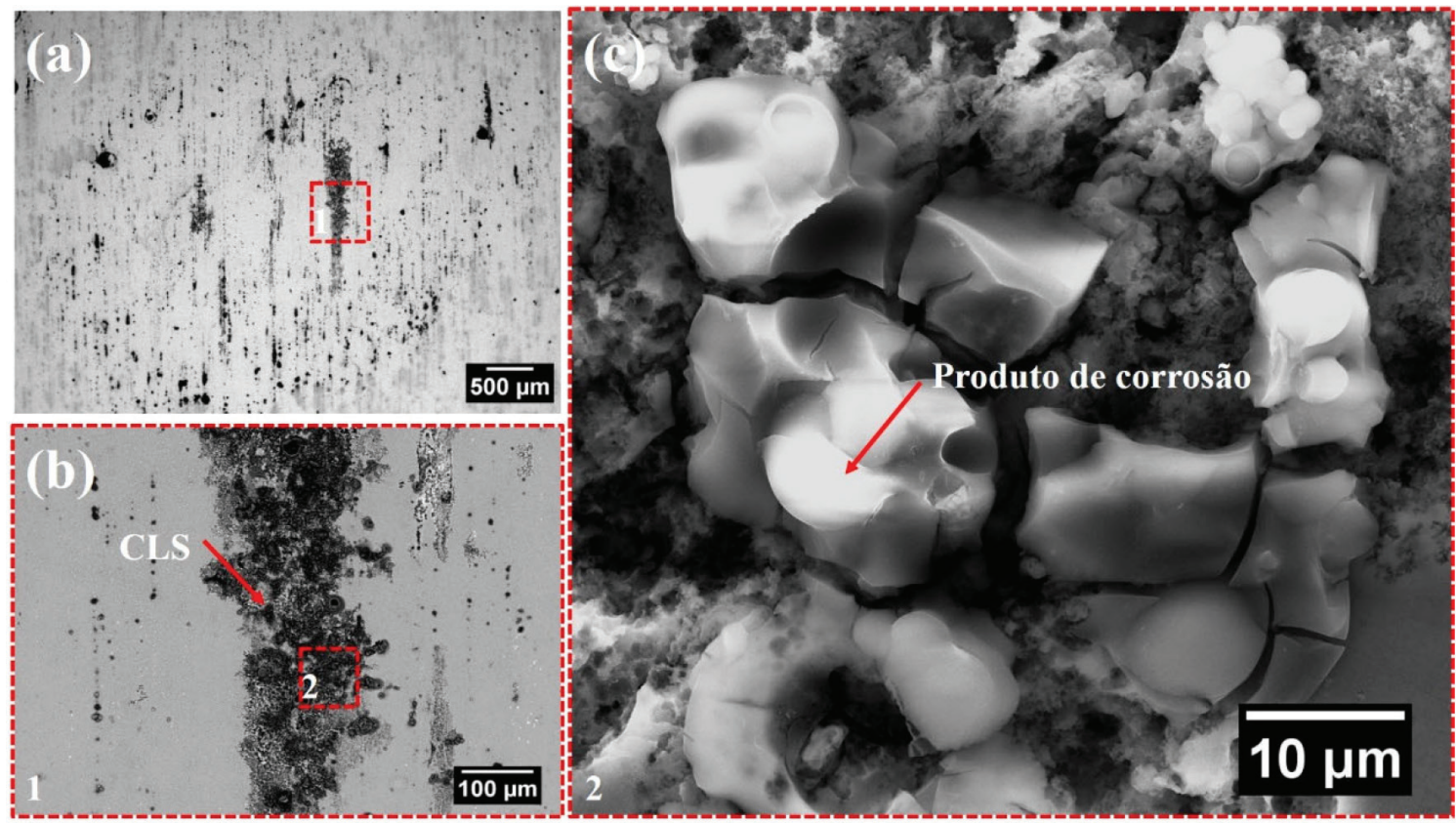

Figura 5. Micrografias obtidas por MEV da liga 2198-T8 após 24 h de imersão em solução contendo 0,01 mol L-1 NaCl: (a) menor magnificação; (b) ampliação do quadrado tracejado em (a) e (c) ampliação do quadrado tracejado em (b)

A evolução de hidrogênio que ocorre desde as primeiras horas de ensaio está associada às regiões anódicas, onde a formação de cátions metálicos promove a hidrólise. Como produto dessa reação, tem-se a liberação de íons $\mathrm{H}^{+}$que diminuem o $\mathrm{pH}$ local. ${ }^{21} \mathrm{O}$ ensaio de gelificação mostrou-se uma técnica simples e eficaz na identificação das regiões anódicas e catódicas, conforme ilustra a Figura 8.

A Figura 8 mostra que nas regiões de CLS, o pH atinge valores muito baixos. A acidificação ocorre principalmente dentro dos pites e resulta na evolução de bolhas de hidrogênio, Figura 7. Devido à baixa mobilidade iônica no teste de gelificação, o pH nas regiões que circundam as regiões de CLS, assume valores muito baixos, como mostra a Figura 9.

Após remoção do gel, as regiões ao redor do sítio anódico se mostraram brilhantes devido à proteção catódica nas áreas vizinhas aos pites de CLS, Figura 6a. As regiões mais distantes da CLS e mais escuras correspondem à parte de coloração verde no gel, onde predomimam a reação redução do oxigênio sobre as partículas micrométricas, resultando um aumento do pH. A CLS exibe características distintas do ataque localizado que é associado às micropilhas galvânicas entre a matriz e as partículas micrométricas, conforme ilustrado anteriormente nas Figuras 4, 5 e 6 . A área onde ocorreu ataque localizado, porém, distribuído de forma generalizada na superfície, apresentou-se escurecida pela formação de produtos de corrosão. A dissolução dos íons de Li e de $\mathrm{Al}$ da fase T1 promove hidrólise resulta na acidificação do meio e propagação da corrosão. A diluição da solução interna ao pite (CLS) é dificultada favorecendo a manutenção da acidificação local, o que é comprovado pela evolução de hidrogênio nestes locais, Figura 6. 

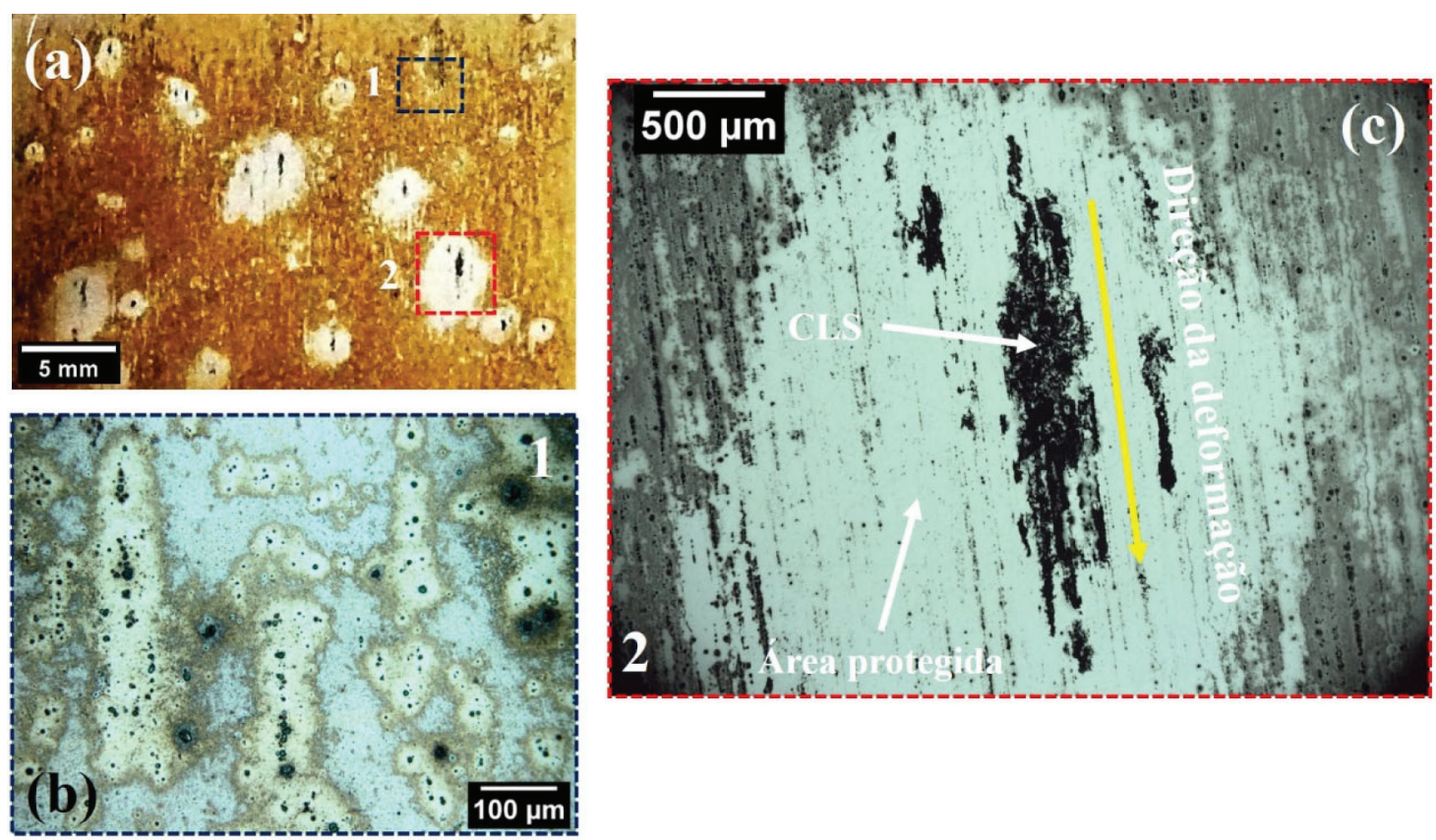

Figura 6. Macrografias da superfície da liga 2198-T8 após 24 h de imersão em solução contendo 0,01 mol $L^{-1} \mathrm{NaCl}$. (a) Visão geral mostrando áreas de corrosão localizada severa (CLS); (b) detalhe da área indicada por 1 na figura (a) mostrando o ataque localizado relacionado com as partículas constituintes $e ;$ (c) detalhe da área indicada por 2 na figura (a) que corresponde a CLS

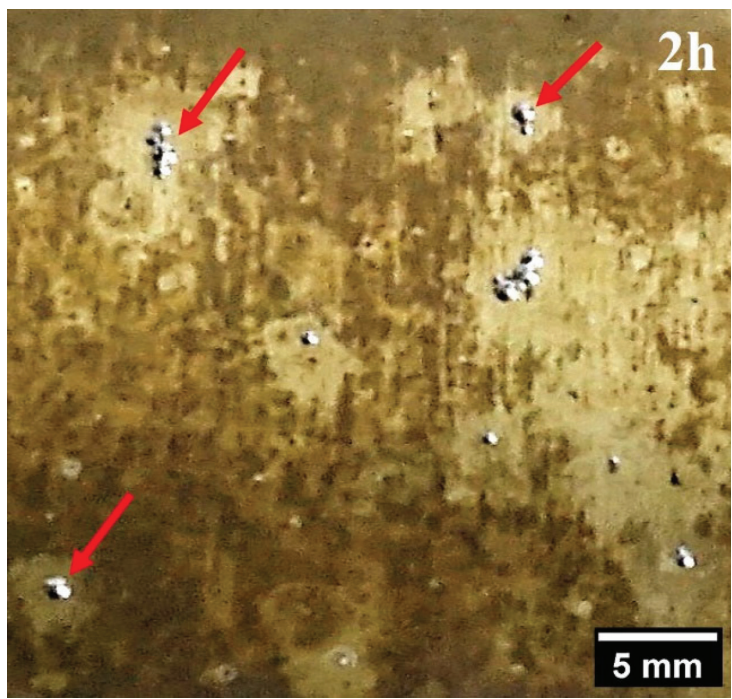

Figura 7. Macrografia da superfície da liga 2198-T8 após 2 h de imersão em solução contendo 0,01 $\mathrm{mol} \mathrm{L}^{-1} \mathrm{NaCl}$. As flechas vermelhas indicam regiões com evolução de gás hidrogênio

Consequentemente, a passivação local é impedida. Deste modo, uma diferença de potencial entre a superfície externa da liga e o interior do pite de CLS é formada, e, consequentemente, a área circundante é protegida catodicamente. As principais reações que envolvem o processo de formação da CLS são apresentadas nas Equações (3) a (6): ${ }^{35}$

$$
\begin{gathered}
\mathrm{Li} \rightarrow \mathrm{Li}^{+}+\mathrm{e}^{-} \\
\mathrm{Al} \rightarrow \mathrm{Al}^{3+}+3 \mathrm{e}^{-} \\
\mathrm{Al}^{3+}+3 \mathrm{H}_{2} \mathrm{O} \rightarrow \mathrm{Al}(\mathrm{OH})_{3}+3 \mathrm{H}^{+} \\
6 \mathrm{H}^{+}+2 \mathrm{Al} \rightarrow 2 \mathrm{Al}^{3+}+3 \mathrm{H}_{2} \uparrow \\
2 \mathrm{H}^{+}+2 \mathrm{e}^{-} \rightarrow \mathrm{H}_{2} \uparrow
\end{gathered}
$$

Durante o processo de corrosão ocorre a oxidação do Li e Al para $\mathrm{Li}^{+}$e $\mathrm{Al}^{3+}$ respectivamente, nas regiões anódicas (3-4), o que promove a hidrólise da água e resulta em diminuição do $\mathrm{pH}$ (5), além de favorecer a evolução do gás hidrogênio (6) e (7). Portanto, a formação de bolhas de hidrogênio é indicativa de $\mathrm{pH}$ baixo e tem sido associada com regiões de CLS. ${ }^{13,14,28}$ Vale destacar que a área catodicamente protegida pode ser vista em escala macroscópica, como mostrou a Figura 6a. A profundidade da penetração da corrosão foi avaliada por perfilometria óptica (PO). Essa técnica foi utilizada com sucesso para investigar as diferenças nas profundidades de ataques por corrosão. ${ }^{36}$ Assim, após o teste de imersão, foram analisados locais de corrosão associados às partículas micrométricas e a CLS, como apresentado na Figura 10.

A profundidade do ataque associado às partículas constituintes variou de 1 a $5 \mu \mathrm{m}$, devido às diferenças nos tamanhos e composição química das partículas micrométricas, ${ }^{28}$ causando assim, variações nas diferenças de potencial entre estas e a matriz, Figura 10a. A profundidade de penetração do ataque associado à CLS variou entre 8 e $20 \mu \mathrm{m}$, Figura 10b. Essa diferença é relacionada à concentração e distribuição da fase $\mathrm{T} 1$, pois, os grãos que apresentaram maior distribuição de fase T1, são aqueles cuja penetração do ataque é maior. ${ }^{28}$ Desta forma, observa-se que o ataque associado à CLS apresenta maior profundidade e, portanto, que este tipo de ataque é mais intenso, com mecanismo de corrosão distinto.

\section{Ensaios eletroquímicos}

Como visto anteriomente, a evolução de hidrogênio observada nas regiões próximas à CLS é um indicativo de baixo $\mathrm{pH}$ e está diretamente relacionada ao desenvolvimento da CLS. Isso foi confirmado pela análise da liga por microscopia eletroquímica de varredura (SECM). Esta técnica permitiu mapear os locais de evolução de hidrogênio durante corrosão da liga e associá-los às regiões de CLS por meio de estudo eletroquímico. As bolhas de hidrogênio geradas próximo aos locais de CLS foram detectadas pelo microeletrodo de Pt polarizado no potencial de $0 \mathrm{~V}$. Nesse potencial ocorre a oxidação do $\mathrm{H}_{2},{ }^{22}$ segundo a Equação 4. Assim, os locais de evolução de hidrogênio podem ser identificados pela técnica SECM, como pode ser observado na Figura 11. 

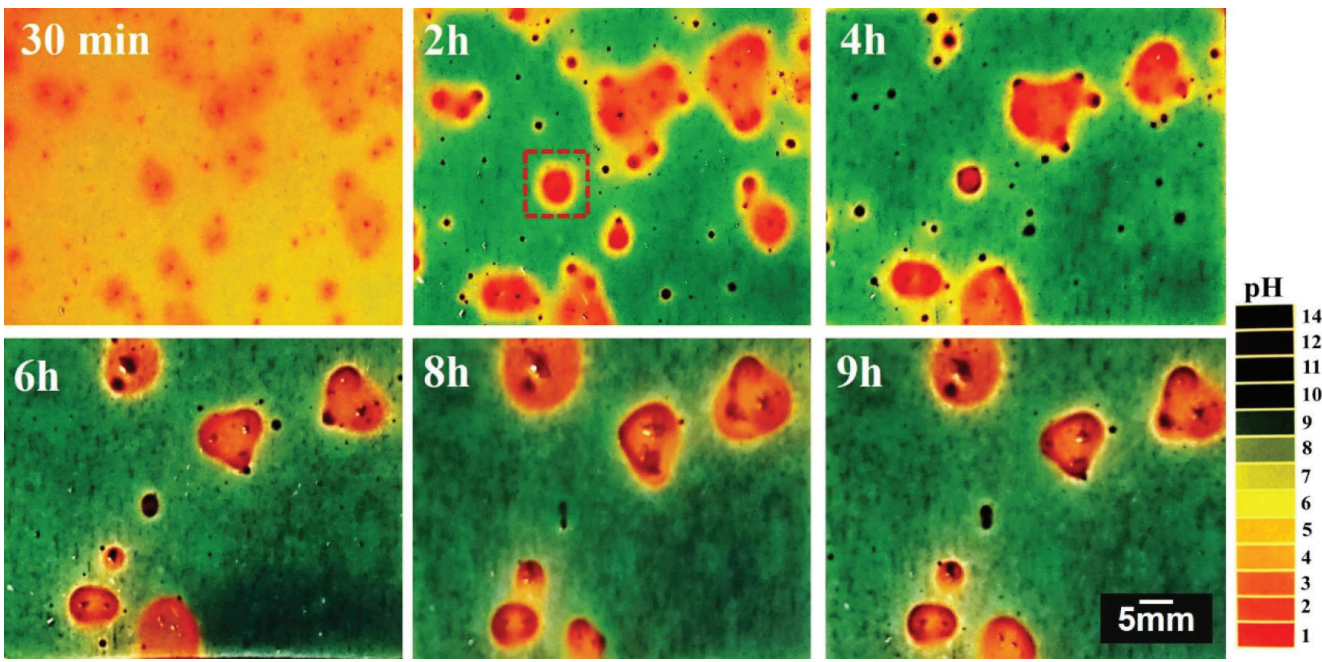

Figura 8. Macrografias da superfície da liga 2198-T8 durante o teste de gelificação

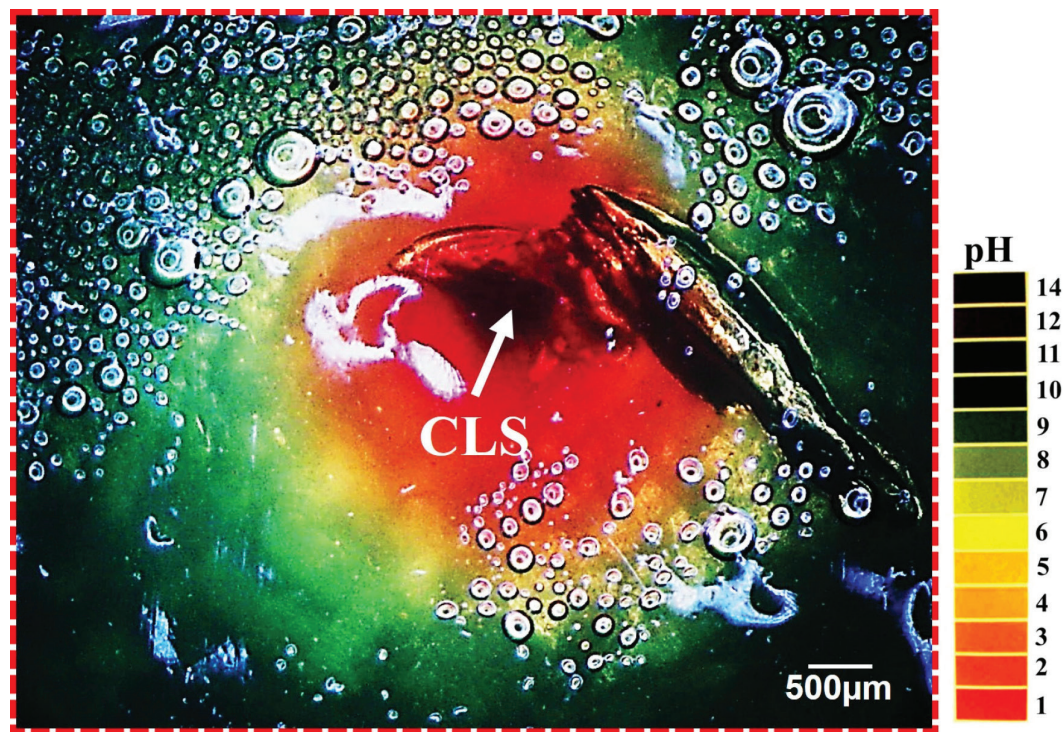

Figura 9. Macrografia em maior ampliação do quadrado tracejado na Figura 8
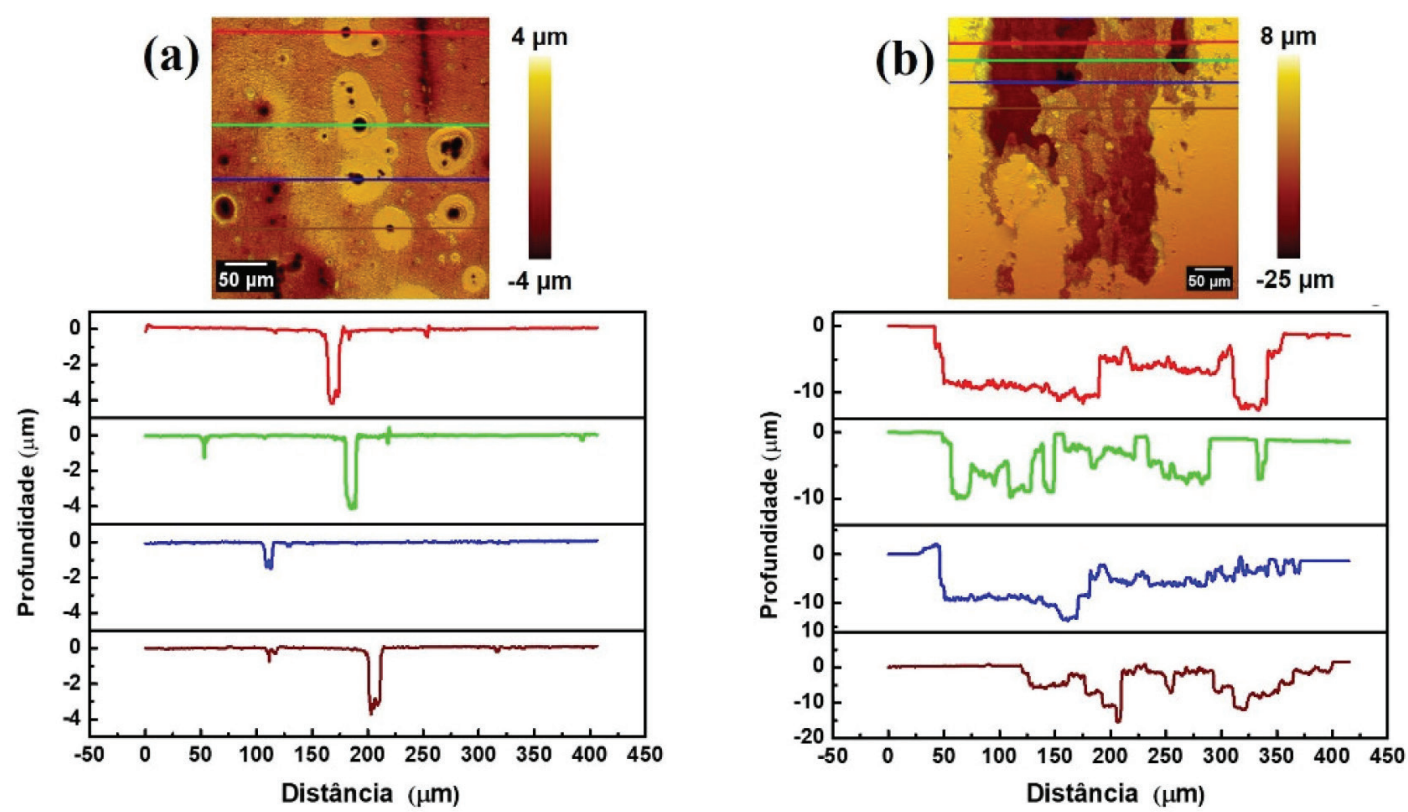

Figura 10. Perfilometria ótica da superfície da liga 2198-T8 após 24 h de imersão em solução contendo 0,01 mol L ${ }^{-1}$ NaCl: (a) ataque associado as partículas micrométricas e (b) ataque associado a CLS 
(a)
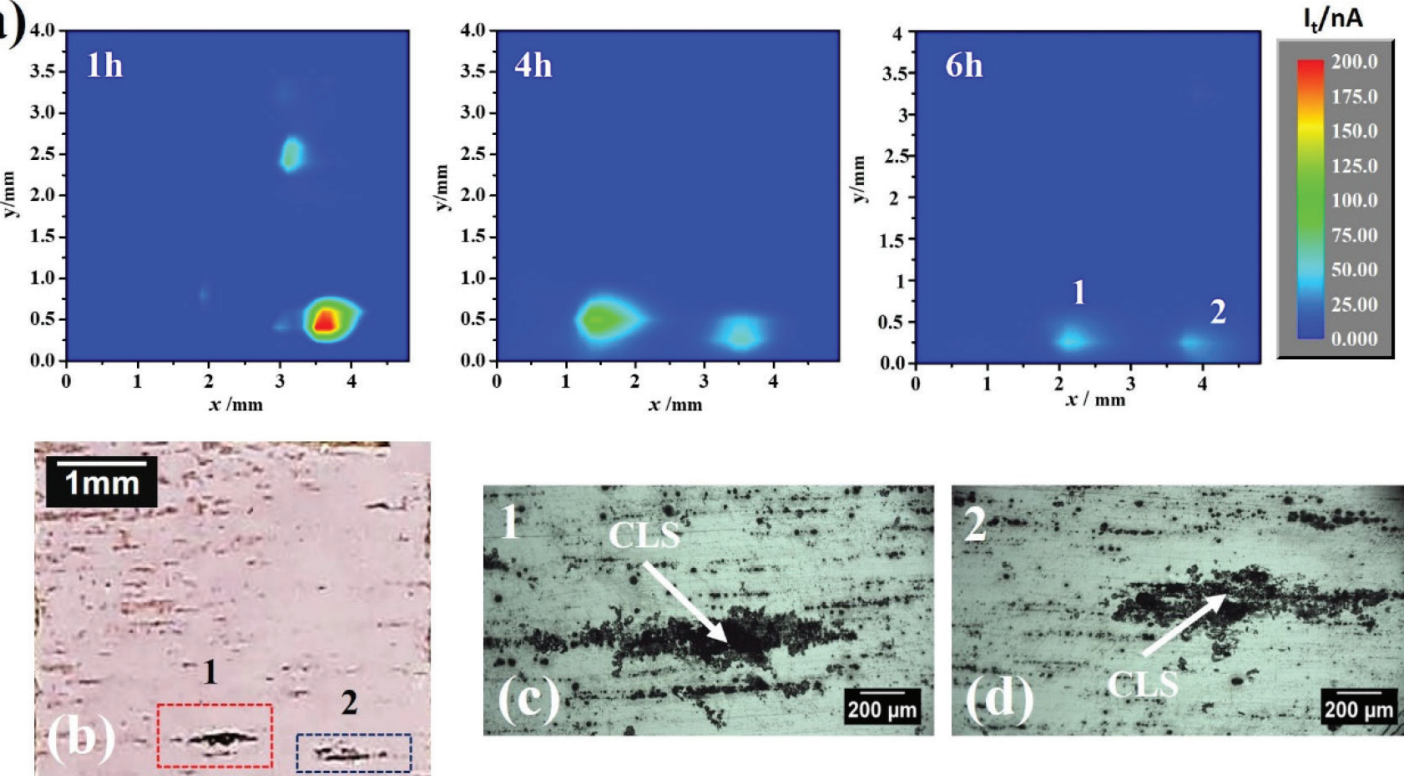

Figura 11. (a) Mapas de SECM da superfície da liga durante exposição à solução contendo 0,01 mol $L^{-1}$ NaCl; (b) superfície da liga após o teste; (c) e (d); locais 1 e 2 associados a CLS indicados nas figuras $(a)$ e (b)

Na Figura 11a a escala de cor fornece uma indicação da intensidade da corrente para a reação de oxidação do hidrogênio gerado na superfície. Após 1 h de imersão, foi detectada evolução de hidrogênio em uma região específica da superficie, e a corrente de oxidação máxima associada foi cerca de $200 \mathrm{nA}$. Após $4 \mathrm{~h}$ de imersão foi identificado novo local de evolução de hidrogênio. No entanto, nesse momento, havia acúmulo de produtos de corrosão na superfície, o que resultou em diminuição no valor máximo da corrente em relação à medida anteriormente obtida. Decorridas $6 \mathrm{~h}$ de imersão, ocorreu diminuição da corrente em ambos locais. A Figura 11b mostra a superfície após o ensaio e os pontos de CLS associadas com a corrente de oxidação gerada. É possível notar que os pontos 1 e 2, identificados nos mapas de SECM, estão associados aos locais de CLS. Nas Figuras 11c e $11 \mathrm{~d}$ é notado o acúmulo de produtos de corrosão, o que fundamenta a hipótese que a obstrução do sítio anódico da CLS é a causa para a diminuição dos valores de corrente no decorrer do ensaio, e, como já observado em outros trabalhos, esse fenômeno interfere nas medidas eletroquímicas locais. ${ }^{10}$

\section{Mecanismo da evolução de gás hidrogênio}

Com base nos resultados obtidos e na literatura, foi proposto um mecanismo de evolução de hidrogênio associado à corrosão localizada severa (CLS), conforme ilustra a Figura 12.

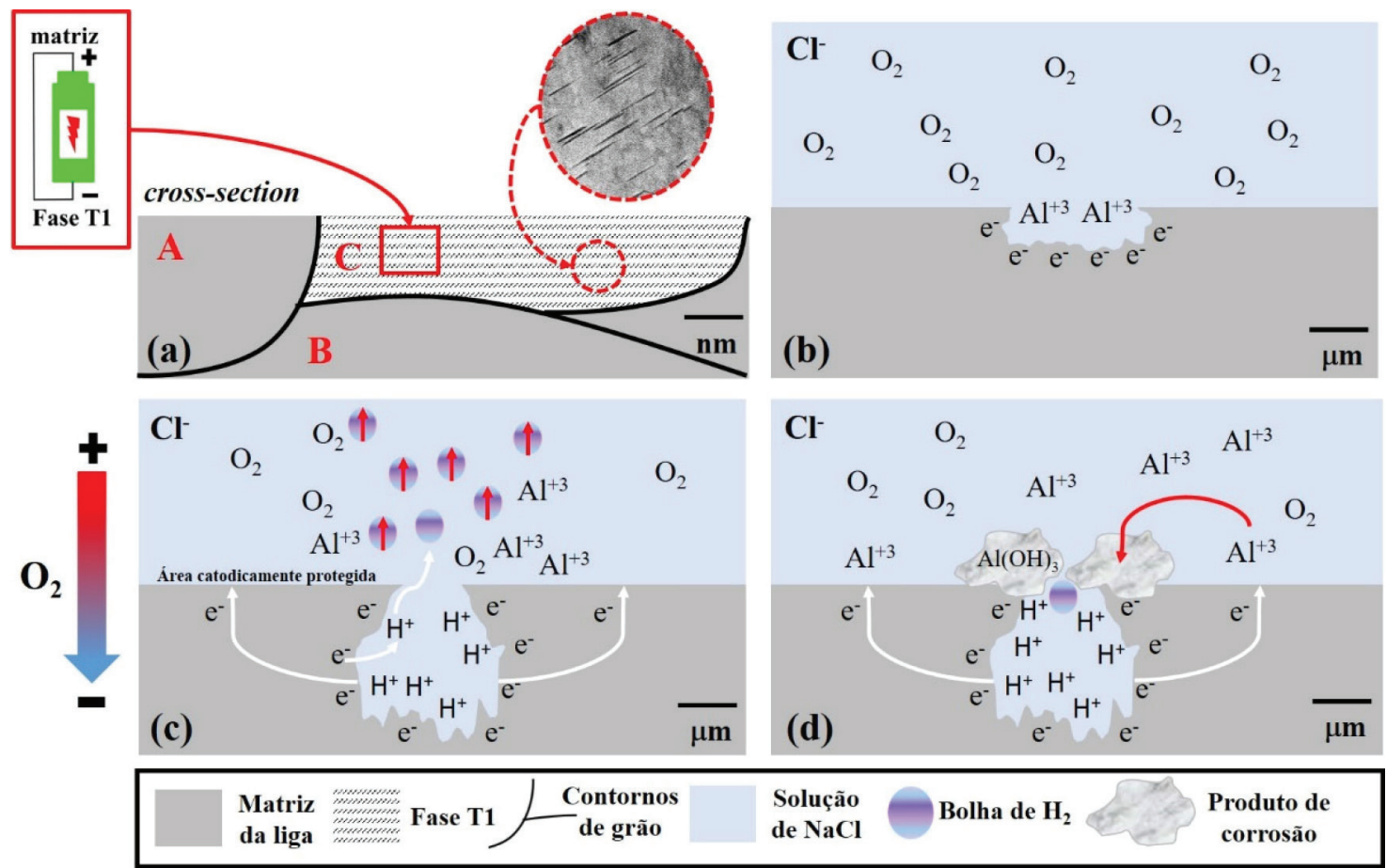

Figura 12. Diagrama esquemático do mecanismo de evolução de $H_{2}$ proposto durante a corrosão da liga 2198-T8: (a) superfície após o polimento; (b), (c) e (d) reações de corrosão durante imersão na solução contendo $0,01 \mathrm{~mol} \mathrm{~L}^{-1} \mathrm{NaCl}$ 
Durante o processo de fabricação das ligas aeronáuticas alguns grãos são mais deformados do que outros, Figura 12a. Assumindo que o grão C é mais deformado do que os grãos A e B, a densidade de fase T1 será maior dentro deste grão e será preferencialmente corroído, pois o par matriz/fase T1 atua como pilha galvânica. A maioria dos elementos ativos na fase $\mathrm{T} 1$ ( $\mathrm{Al}$ e Li) são preferencialmente dissolvidos, ocorrendo o enriquecimento em $\mathrm{Cu}$, e, consequentemente, a inversão de polaridade com a matriz da liga que passa a ser atacada, ${ }^{37}$ Figura 12b. Consequentemente, a matriz é corroída e os íons $\mathrm{Al}^{+3}$ são gerados dentro do pite, Figura $12 \mathrm{~b}$. A penetração da CLS atinge elevada profundidade, como mostrado na Figura 9. Dentro do pite, a presença dos íons $\mathrm{Al}^{+3}$ promovem a hidrólise da água, e os íons $\mathrm{H}^{+}$ em excesso causam a diminuição local do $\mathrm{pH}$. A redução de íons $\mathrm{H}^{+}$ dentro dos locais de CLS resulta na formação de bolhas de hidrogênio. Na parte externa do pite ocorre precipitação de produtos de corrosão os quais dificultam a entrada de oxigênio, causando uma diferença de aeração da parte interna do pite a parte externa, e a mistura do meio externo com o meio interno, o que favorece a continuação do processo de corrosão, como foi observado no ensaio de gelificação, Figuras 8 e 9. As regiões vizinhas ao pite correspondem às áreas catodicamente protegidas. Todos esses fenomênos são ilustrados na Figura 12c. Na parte externa do pite, ocorre a redução do $\mathrm{O}_{2}$ produzindo $\mathrm{OH}^{-}$, os quais, ao se combinarem com os íons $\mathrm{Al}^{3+}$, causam a precipitação do produto de corrosão $\mathrm{Al}(\mathrm{OH})_{3}$, como mostrado na Figura 3c, nas áreas do entorno do pite, Figura 12d. O acúmulo de produtos de corrosão na entrada do pite e, consequentemente, a obstrução da entrada dele, dificulta a evolução do $\mathrm{H}_{2}$. Isso explica o aumento do $\mathrm{pH}$ no decorrer do teste de gelificação e a diferença de sinal medido pelo microeletrodo de SECM no decorrer do ensaio, devido à formação dos produtos de corrosão, Figura 11a.

\section{CONCLUSÃO}

A evolução de gás hidrogênio na liga 2198-T8 está diretamente associada ao desenvolvimento da corrosão localizada severa (CLS), em consequência do ataque à fase T1. Ela tem início nos grãos mais ativos eletroquimicamente e sua morfologia foi diretamente relacionada à microestrutura da liga. $\mathrm{O}$ acúmulo de produtos de corrosão nos locais relacionados a CLS tem importante papel no processo de corrosão, dificultando a evolução das bolhas de $\mathrm{H}_{2}$ e, consequentemente, interferindo nas medidas eletroquímicas locais, como observado por SECM. Os testes de imersão e visualização em gel mostraram que a cinética de evolução do gás hidrogênio é rápida desde as primeiras horas de imersão. A técnica SECM mostrou-se útil para monitorar os locais de evolução de hidrogênio desenvolvidos durante a corrosão espontânea de ligas de Al-Cu-Li.

\section{AGRADECIMENTOS}

Os autores agradecem à FAPESP (Processos 2019 / 18388-1 e 2018 / 06880-6) pelo apoio financeiro a esta pesquisa.

\section{REFERÊNCIAS}

1. Vargel, C.; Jacques, M.; Schmidt, M. P.; Corrosion of Aluminium, $1^{\text {st }}$ ed., Elsevier: Oxford, 2004.

2. Birbilis, N.; Buchheit, R. G. J.; J. Electrochem. Soc. 2005, 152, 140.

3. Revie, R. W.; Uhlig, H.; Corrosion and Corrosion Control, $1^{\text {st }}$ ed., John Wiley \& Sons: EUA, 2008.

4. Pieretti, E. F.; Manhabosco, S. M.; Dick, L. F. P.; Hinder, S.; Costa, I.; Electrochim. Acta 2014, 124, 150.

5. Becker, A.; Mater. Corros. 2002, 53, 560.
6 Sun, P.; Wang, Z.; Lu, Y.; Shen, S.; Yang, R.; Xue, A.; Parker, T.; Wang, J.; Wang, Q.; Process Saf. Environ. Prot. 2020, 135, 244.

7. Rioja, R. J.; Liu, J.; Metall. Mater. Trans. A 2012, 43, 3325.

8. Wanhill, R. J. H.; Aerospace Applications of Aluminum-Lithium Alloys, $1^{\text {st }}$ ed., Elsevier: Oxford, 2013.

9. Milagre, M. X.; Mogili, N. V.; Donatus, U.; Giorjão, R. A. R.; Terada, M.; Araujo, J. V. S. U.; Machado, C. S. C.; Costa, I.; Mater. Charact. 2018, 140, 233.

10. de Sousa Araujo, J. V.; Donatus, U.; Queiroz, F. M.; Terada, M.; Milagre, M. X.; Alencar, M.C.; Costa, I.; Corros. Sci. 2018, 133, 132.

11. Ma, Y.; Zhou, X.; Liao, Y.; Yi, Y.; Wu, H.; Wang, Z.; Huang, W.; Corros. Sci. 2016, 107, 41.

12. Donatus, U.; Berbel, L. O.; Costa, I.; Mater. Corros. 2018, 69, 1375.

13 Chen, B.; Li, C. H.; He, S.C.; Li, X. L.; Lu, C.; Li, C. H.; He, S. C.; Li, X. L.; Lu, C.; J. Mater. Res. 2014, 29, 1344.

14. Ma, Y.; Zhou, X.; Huang, W.; Thompson, G.E.; Zhang, X.; Luo, C.; Sun, Z.; Mater. Chem. Phys. 2015, 161, 201.

15. Lei, X.; Saatchi, A.; Ghanbari, E.; Dang, R.; Li, W.; Wang, N.; Macdonald, D. D.; Materials 2019, 12, 1600.

16. Queiroz, F. M.; Donatus, U.; Ramirez, O. M. P.; Araujo, J. V. S.; Viveiros, B. V. G.; Lamaka, S.; Zheludkevich, M.; Masoumi, M.; Vivier, V.; Costa, I.; Melo, H. G.; Electrochim. Acta 2019, 313, 271.

17. Benedyk, J. C.; Light Metal Age, $4^{\text {th }}$ ed., Light Metal Age Magazine: EUA, 2010.

18. Dorin, T.; Vahid, A.; Lamb, J.; Fundamentals of Aluminium Metallurgy, $1^{\text {st }}$ ed., Elsevier: Oxford, 2018.

19. Coutinho, T. A.; Metalografia de Não Ferrosos: Análise e Prática, $1^{\mathrm{a}} \mathrm{ed}$., Edgard Blucher: Brazil, 1980.

20. Davis, J. R.; Metals Handbook, 1 ${ }^{\text {st }}$ ed., CRC Press: EUA, 1973.

21. Isaacs, H. S.; Adzic, G.; Jeffcoate, C. S.; Corrosion 2000, 56, 971.

22. Bard, A. J.; Fan F. R. F.; Kwak, J.; Lev, O.; Anal. Chem. 1989, 61, 132.

23. Souto, R. M.; Lamaka, S. V.; González, S.; González, Microsc. Sci. Technol. Appl. Educ. 2010, 3, 1769.

24. Santana, J. J.; González, S.; Izquierdo, J.; Souto, R. M.; Afinidad 2011, $68,44$.

25. Senöz, C.; Maljusch, A.; Rohwerder, M.; Schuhmann, W.; Electroanalysis 2012, 24, 239.

26. Gupta, R. K.; Nayan, N.; Nagasireesha, G.; Sharma, S. C.; Mater. Sci. Eng. A 2006, 420, 228.

27. Zhou, Y.; Sun, K.; Frankel, G. S.; J. Electrochem. Soc. 2018, 165, 807.

28. Araujo, J.V. S.; Bugarin, A. F. S.; Donatus, U.; Machado, C.S.C.; Queiroz, F.M.; Terada, M.; Astarita, A.; Costa, I.; Corros. Eng. Sci. Technol. 2019, 54, 575.

29. Ma, Y.; Zhou, X.; Thompson, G.E.; Hashimoto, T. P.; Fowles, T. M., Mater. Chem. Phys. 2011, 126, 46

30. Grilli, R.; Baker, M. A.; Castle, J. E.; Dunn, B.; Watts, J. F.; Corros. Sci. 2010, 52, 2855.

31. Buchheit, R. G.; Moran, J. P.; Stoner, G. E.; Corrosion 1990, 46, 610.

32. Pedeferri, P.; Corrosion Science and Engineering, $1^{\text {st }}$ ed., Springer: Switzerland, 2019.

33. Zhou, X.; Luo, C.; Ma, Y.; Hashimoto, T.; Thompson, G. E.; Hughes, A. E.; Skelson, P.; Surf. Interface Anal. 2013, 45, 1543.

34. Donatus, U.; Terada, M.; Ospina, C. R.; Queiroz, F. M.; Bugarin, A. F. S.; Costa, I.; Corros. Sci. 2018, 131, 300.

35. Luo, C.; Albu, S. P.; Zhou, X.; Sun, Z.; Zhang, X.; Tang, Z.; Thompson, G. E.; J. Alloys Compd. 2016, 658, 61.

36. Ghosh, R.; Venugopal, A.; Narayanan, P. R.; Sharma, S. C.; Venkitakrishnan, P. V.; Trans. Nonferrous Met. Soc. China 2017, 24, 241.

37. Li, J. F.; Li, C. X.; Peng, Z. W.; Chen, W. J.; Zheng, Z. Q.; J. Alloys Compd. 2008, 460, 688. 